\title{
Avaliação do Conhecimento de Estudantes de Medicina sobre Morte Encefálica*
}

\section{Evaluation of Medical Students Knowledge on Brain Death}

\author{
Almir Galvão Vieira Bitencourt ${ }^{1,2}$, Flávia Branco Cerqueira Serra Neves ${ }^{2,3}$, Larissa Durães 2,3, $^{2,3}$ \\ Diego Teixeira Nascimento ${ }^{1,2}$, Nedy Maria Branco Cerqueira Neves ${ }^{4}$, Lara de Araújo Torreão ${ }^{5}$, Sydney Agareno ${ }^{6}$
}

\section{RESUMO}

JUSTIFICATIVA E OBJETIVOS: Por ser um conceito relativamente novo e pouco divulgado na sociedade, o diagnóstico de morte encefálica (ME) ainda não é bem aceito pela população em geral, inclusive entre médicos e estudantes de Medicina. O objetivo deste estudo foi avaliar o conhecimento de uma amostra de estudantes de Medicina sobre o protocolo diagnóstico de ME.

MÉTODO: Estudo descritivo de corte transversal, avaliando acadêmicos de duas faculdades de Medicina de Salvador-BA. Foi distribuído um questionário auto-aplicável composto por questões referentes à conhecimento, técnico e ético, contidos na Resolução n 1.480/97 do Conselho Federal de Medicina, que dispõe sobre os critérios para caracterização de ME.

1. Graduando da Faculdade de Medicina da Bahia (UFBA).

2. Membro da Liga Acadêmica de Medicina da Bahia (LAMIB).

3. Graduando da Escola Bahiana de Medicina e Saúde Pública.

4. Médica Oftalmologista, Professora de Ética Médica e Bioética da Escola Bahiana de Medicina e Saúde Pública, Mestre em Educação (FACED-UFBA); Doutoranda em Medicina (PPGMS-UFBA).

5. Médica Pediatra, Coordenadora da UTI Pediátrica do Hospital Aliança e do Hospital Ernesto Simões Filho, Mestre em Pediatria (FMUSP).

6. Médico Intensivista, Coordenador do Projeto Morte Encefálica da Associação de Medicina Intensiva Brasileira - AMIB, Membro Consultor da Liga Acadêmica de Medicina Intensiva da Bahia.

${ }^{*}$ Recebido da Liga Acadêmica de Medicina Intensiva da Bahia (LAMIB), Salvador, BA

Apresentado em 09 de março de 2007

Aceito para publicação em 23 de abril de 2007

Endereço para correspondência:

Almir Galvão Vieira Bitencourt

Rua Vanderlei Pinho, 181/802 - Itaigara

41815-270 Salvador, BA

Fone: (71) 8806-0515 / 3451-4063

E-mail: almirgvb@yahoo.com.br

(C)Associação de Medicina Intensiva Brasileira, 2007
RESULTADOS: Foram avaliados 115 estudantes. A média de acertos nas 14 questões sobre o conhecimento dos critérios da ME foi de 6,7 $\pm 1,8$; sendo maior entre os estudantes que haviam assistido alguma apresentação sobre ME. A maioria dos estudantes $(87,4 \%)$ soube identificar os pacientes candidatos ao protocolo de ME. No entanto, apenas 5,2\% e 16,1\% dos estudantes acertaram, respectivamente, os testes clínicos e complementares que devem ser realizados durante o protocolo. Frente a um paciente não-doador com diagnóstico confirmado de ME, 66,4\% referiram que o suporte artificial de vida deve ser suspenso. Apenas 15\% dos estudantes entrevistados já avaliaram um paciente com ME, sendo este percentual maior entre os que já haviam realizado estágio em UTI $(38,2 \%$ versus $5,1 \%$; $\mathrm{p}<0,001)$.

CONCLUSÕES: Os resultados deste estudo apontaram para um conhecimento limitado dos estudantes avaliados sobre os critérios para caracterização da ME, principalmente em relação à sua abordagem prática.

Unitermos: educação médica, estudantes de Medicina, morte encefálica, transplante de órgãos.

\section{SUMMARY}

BACKGROUND AND OBJECTIVES: Because brain death $(\mathrm{BD})$ is a new concept and little divulged, it's not well accepted in general population, including doctors and Medical students. This study aims to evaluate the knowledge of a sample of Medical students on the Brazilian BD diagnosis protocol.

METHODS: Descriptive cross-sectional survey that evaluated students from two medical schools in Salvador-BA. We used a questionnaire composed by questions about technical and ethical knowledge contained in the Federal Council of Medicine's Resolution $n^{\circ}$ 1480/97 that establishes the criteria for BD diagnosis. RESULTS: We evaluated 115 Medical students. In 14 questions about the knowledge of BD criteria, the 
mean of right answers were $6.7 \pm 1.8$, which were higher among the students that had attended some presentation on BD. Most of the students (87.4\%) knew how to identify the candidates to the BD diagnosis protocol. However, only $5.2 \%$ and $16.1 \%$ of the students answered right, respectively, the clinical and complementary tests that should be accomplished during the diagnosis protocol. Facing a no-donor patient with confirmed diagnosis of BD, $66.4 \%$ referred that artificial life support should be suspended. Only $15 \%$ of the interviewed students had already evaluated a patient with $\mathrm{BD}$, being this percentage higher among those who had already frequented ICU $(38.2 \%$ versus $5.1 \%$; $\mathrm{p}<0.001$ ).

CONCLUSIONS: The results of this study showed a limited knowledge of the evaluated students on BD diagnosis criteria, mainly in relation to the practical approach of this condition.

Key Words: brain death, Medical education, Medical students, organ transplantation.

\section{INTRODUÇÃO}

A morte encefálica (ME) é definida como parada completa e irreversível das funções encefálicas, impossibilitando a manutenção da vida sem o auxílio de meios artificiais" . Os critérios para o diagnóstico de ME, estabelecidos a partir da década de 1960, envolvem parâmetros clínicos e, em alguns países, inclusive o Brasil, a realização de exames complementares. A ME deverá ser conseqüência de processo irreversível e de causa conhecida que demonstrem, durante um determinado intervalo de tempo, de forma inequívoca, ausência da perfusão sangüínea cerebral ou da atividade elétrica ou da atividade metabólica². Apesar deste conceito já estar bem definido na comunidade científica mundial há pelo menos 30 anos, ele ainda não é bem aceito pela população em geral, inclusive entre médicos e estudantes de Medicina ${ }^{3,4}$.

A não aceitação da morte, sendo este ainda um paradigma para a sociedade, tem dificultado a definição dos limites terapêuticos nos cuidados intensivos, contrapondo os princípios éticos e bioéticos. Também é comum a confusão entre os conceitos de $\mathrm{ME}$ e de paciente terminal, sendo importante destacar que o primeiro está morto e, portanto, a suspensão das medidas de suporte de vida, nesse caso, pode ser realizada sem prejuízo à autonomia do paciente ou da família. Por estes motivos, suspeitar quando um paciente pode estar em ME, confirmar este diagnóstico e conduzir estes casos é fundamental não só para melhorar a alo- cação de órgãos para transplante, como também para evitar a manutenção artificial da vida em pacientes que já estão mortos. Estudo realizado recentemente pela Sociedade de Terapia Intensiva da Bahia (SOTIBA), com o apoio da Associação de Medicina Intensiva Brasileira (AMIB), evidenciou que o conhecimento acerca dos critérios de ME é ainda pouco difundido entre os médicos intensivistas ${ }^{5}$. A desinformação quanto aos aspectos éticos e legais deste diagnóstico gera atitudes incoerentes e contribui para a realização de intervenções desnecessárias em pacientes mortos.

Alguns trabalhos na literatura que buscaram avaliar o conhecimento dos estudantes de Medicina sobre o diagnóstico de ME também mostraram que ele é limitado $^{6-12}$. No entanto, estes trabalhos tinham como objetivo principal avaliar o conhecimento e a opinião de estudantes de Medicina sobre doação e transplante de órgãos. Desta forma, estudos que explorem especificamente os critérios para caracterização da ME podem melhor determinar o nível de conhecimento dos estudantes sobre este tema.

O objetivo deste estudo foi avaliar o conhecimento de uma amostra de estudantes de Medicina de SalvadorBA sobre o protocolo diagnóstico de $\mathrm{ME}$, com base na Resolução n $1480 / 97$ do CFM.

\section{MÉTODO}

Foi realizado um estudo descritivo, de corte transversal, no qual foram avaliados acadêmicos de Medicina de diversos semestres de duas faculdades de Salvador-BA, uma pública (Faculdade de Medicina da Bahia da Universidade Federal da Bahia) e outra privada (Escola Bahiana de Medicina e Saúde Pública). A coleta dos dados foi feita durante a realização do $1^{\circ}$ Simpósio da Liga Acadêmica de Medicina Intensiva da Bahia (LAMIB), em 15 de setembro de 2006, evento realizado com o objetivo de divulgar temas relacionados à Medicina Intensiva na população acadêmica. Foi distribuído um questionário auto-aplicável aos estudantes presentes no início do evento. O questionário foi dividido em duas partes: a primeira, com dados demográficos e relativos à graduação, estágios extracurriculares em unidade de terapia intensiva (UTI) e se haviam assistido alguma apresentação prévia sobre ME; a segunda parte composta por 22 questões objetivas, sendo 14 referentes ao conhecimento sobre o protocolo de ME e seis sobre opiniões a respeito do tema.

O questionário foi baseado em modelo utilizado pela SOTIBA/AMIB em estudo realizado para avaliação do 
conhecimento dos médicos intensivistas sobre $\mathrm{ME}^{5}$. Este questionário foi adaptado pelos autores para aplicação aos estudantes de medicina. As questões relativas ao protocolo de ME foram baseadas na Resolução $\mathrm{n}^{\circ}$ 1.480/97 do Conselho Federal de Medicina (CFM) que define os critérios para o diagnóstico de ME no Brasil. Estas questões se referiram às diversas etapas do processo, como indicação de abertura do protocolo, quem deve fazer as avaliações, intervalo entre as avaliações, exames clínicos e complementares que devem ser realizados e condutas a serem tomadas em diversos momentos durante o protocolo. Os alunos opinaram ainda, a respeito da importância de se realizar o diagnóstico de ME, os fatores que dificultam este diagnóstico, se haviam participado, em algum momento, do protocolo de ME e sobre doação de órgãos para transplantes.

A participação no estudo foi voluntária e sigilosa sem identificação dos alunos que responderam o questionário. Além disso, nas instruções havia um termo de consentimento sobre a participação no estudo e publicação dos dados. Os questionários foram recolhidos imediatamente antes da palestra sobre ME que ocorreu no evento. Foram distribuídos 200 questionários, dos quais 115 foram respondidos.

A análise dos dados foi feita através do programa Statistical Packcage for Social Science (SPSS) na versão 9.0. Foram utilizados os parâmetros da estatística descritiva adotando-se as medidas usuais de tendência central e de dispersão, e cálculos de freqüências simples e relativas. Para a correlação entre as variáveis foram utilizados os testes Qui-Quadrado para as freqüências das variáveis categóricas; $t$ de Student, para as variáveis contínuas com distribuição normal e MannWhitney para as variáveis contínuas sem distribuição normal. O nível significativo adotado foi de $5 \%$.

\section{RESULTADOS}

Foram avaliados 115 questionários. A idade média dos estudantes avaliados foi de 22,5 $\pm 1,5$ anos, variando de 18 a 27 anos. Outras características demográficas dos estudantes estão ilustradas na tabela 1. A maioria já havia assistido alguma apresentação sobre $\mathrm{ME}$ (71,3\%), destes $64,3 \%$ tiveram alguma aula sobre o tema na faculdade, principalmente nas disciplinas de clínica cirúrgica na escola pública e ética médica e bioética na escola privada e $11,3 \%$ assistiram palestras em congressos, simpósios e afins. Menos da metade dos alunos avaliados referiu estágio prévio em
UTI $(43,5 \%)$ e apenas um deles $(0,9 \%)$ o fez em atividades curriculares, enquanto $22,6 \%$ freqüentaram UTI em atividade extracurricular de pesquisa e $30,4 \%$ em atividade extracurricular de plantão.

Tabela 1 - Características Demográficas dos Estudantes Avaliados $(n=115)$.

\begin{tabular}{lc}
\hline Variáveis & $\%(\mathrm{n})$ \\
\hline Sexo feminino & $59,8 \%(67)$ \\
Faculdade pública & $52,2 \%(59)$ \\
Ano de curso & \\
$1^{\circ}$ ano & $3,5 \%(4)$ \\
$2^{\circ}$ ano & $9,6 \%(11)$ \\
$3^{\circ}$ ano & $14,8 \%(17)$ \\
$4^{\circ}$ ano & $41,7 \%(48)$ \\
$5^{\circ}$ ano & $22,6 \%(26)$ \\
$6^{\circ}$ ano & $7,9 \%(9)$ \\
Religião & \\
Católica & $64,6 \%(73)$ \\
Espírita & $10,6 \%(12)$ \\
Evangélica & $7,1 \%(08)$ \\
Sem religião & $17,7 \%(20)$ \\
\hline
\end{tabular}

A média de acertos nas 14 questões referentes ao conhecimento sobre o protocolo de ME foi de 6,7 $\pm 1,8$, com o mínimo de três e o máximo de 11 acertos. Não houve relação entre o número de acertos e o sexo ou o fato de já ter freqüentado UTI. Os estudantes que haviam assistido alguma apresentação sobre ME apresentaram maior média de acertos $(7,0 \pm 1,7$ versus $6 \pm$ 1,$7 ; p=0,009$ ).

A maioria dos estudantes $(87,4 \% ; n=97)$ soube identificar corretamente os pacientes que seriam candidatos ao protocolo de ME, ou seja, todo paciente em coma de causa conhecida, com índice de coma de Glasgow 3 , sem reflexos do tronco e em apnéia. Em relação à participação do médico no protocolo de diagnóstico da ME, 90,6\% ( $n=104)$ dos estudantes responderam corretamente que o médico da central de transplantes não deve participar do processo diagnóstico e $91 \%$ (n = 101) que um neurologista deve sempre realizar um dos exames clínicos. Quanto ao intervalo de tempo mínimo entre os dois exames clínicos do protocolo em pacientes maiores de 2 anos de idade, foi respondido corretamente por $66,1 \%(n=74)$ dos estudantes como seis horas.

Apenas 5,2\% $(n=6)$ dos estudantes acertaram todos os testes propedêuticos que devem ser realizados na avaliação clínica de pacientes com suspeita de ME, enquanto $73,9 \%(n=85)$ deixaram de assinalar algum exame correto e $60 \%(n=69)$ assinalaram algum exame errado. A tabela 2 mostra todos os testes clínicos 
que foram questionados e o percentual de respostas de cada um. Em relação aos exames complementares, apenas $16,1 \%(n=18)$ responderam corretamente os exames que podem ser realizados dentre os presentes na questão (Tabela 3). Quando questionados sobre a necessidade de repetição ou realização de outro exame (em pacientes maiores de 2 anos de idade) após confirmação do diagnóstico de ME com um exame complementar, a maioria dos estudantes respondeu afirmativamente $(67,3 \% ; n=76)$.

Tabela 2 - Testes Propedêuticos que devem ser Realizados durante a Avaliação Clínica de Pacientes com Suspeita de Morte Encefálica e a Freqüência de Respostas Afirmativas.

\begin{tabular}{lc}
\hline Testes & $\%(\mathrm{n})$ \\
\hline Coma aperceptivo & $48,7(56)$ \\
Pupilas fixas e arreativas & $88,7(102)$ \\
Sinal de Babinski * & $39,1(45)$ \\
Ausência de reflexo córneo palpebral & $78,3(90)$ \\
Ausência de reflexo óculo-cefálico & $74,8(86)$ \\
Ausência de resposta às provas calóricas & $39,1(45)$ \\
Ausência de reflexos medulares * & $49,6(57)$ \\
Ausência de reflexo da tosse & $60,9(70)$ \\
Ausência de reflexos patelares * & $25,2(29)$ \\
Apnéia & $83,5(96)$ \\
\hline
\end{tabular}

*Estes testes não fazem parte dos critérios para o diagnóstico de ME.

Tabela 3 - Exames Complementares que devem ser Realizados para Confirmação do Diagnóstico de Morte Encefálica e a Freqüência de Respostas Afirmativas.

\begin{tabular}{lc}
\hline Exames complementares & $\%(\mathrm{n})$ \\
\hline Arteriografia & $45,5(51)$ \\
Eletroencefalograma (EEG) & $92,0(103)$ \\
Estudo do líquor * & $5,4(6)$ \\
Doppler transcraniano & $55,4(62)$ \\
Tomografia simples de crânio * & $17,9(20)$ \\
\hline
\end{tabular}

*Estes exames não devem ser utilizados para o diagnóstico de ME.

Grande parte dos estudantes $(80,7 \% ; n=88)$ sabia que a notificação da ME é compulsória e praticamente todos $(99,1 \% ; n=112)$ concordaram que este diagnóstico pode ser feito em pacientes não-doadores. No entanto, frente a um paciente não-doador em que foi confirmado o diagnóstico de ME, apenas $66,4 \%$ ( $n=$ 75) referiram que o suporte artificial de vida deve ser suspenso nesses casos.

Os alunos foram questionados sobre qual atitude tomar frente ao seguinte caso clínico: "Um paciente de 35 anos, vítima de acidente automobilístico com traumatismo cranioencefálico (TCE) grave é admitido na UTI, onde é confirmado o diagnóstico de ME. Apesar do médico assistente ter acompanhado todo o protocolo de diagnóstico, a família não aceita que os aparelhos sejam desligados, bem como contrária à doação dos órgãos, apesar do paciente ter declarado, em vida, ser doador". Apenas 16,2\% ( $n=18)$ dos estudantes afirmaram que o médico deve desligar os aparelhos neste caso, enquanto que 49,5\% ( $n=55)$ acharam que é obrigatório ou prudente respeitar a família, 31,5\% $(n=35)$ acharam que deve haver um consenso entre a equipe médica e a família e 2,7\% $(n=3)$ que o médico deve iniciar os procedimentos para doação dos órgãos independente da vontade da família.

Três questões sobre o conhecimento do protocolo de $\mathrm{ME}$ foram relativas às condutas a serem tomadas pelo médico plantonista antes de iniciar o protocolo, entre o $1^{\circ}$ e $\circ 2^{\circ}$ exames clínicos e após a confirmação do diagnóstico. As condutas que foram questionadas e o percentual de resposta dos estudantes para cada uma delas estão ilustrados na tabela 4.

Tabela 4 - Condutas a serem Tomadas em Diversos Momentos durante o Protocolo de Morte Encefálica

\begin{tabular}{lc}
\hline Condutas no início do protocolo de ME & $\%(\mathrm{n})$ \\
Comunicar ao médico assistente & $73,2(84)$ \\
Acionar a família para possível doação & $65,8(75)$ \\
Comunicar a central de órgãos & $48,2(55)$ \\
\hline Condutas entre o $1^{\circ}$ e o $2^{\circ}$ exames clínicos & $\%(\mathrm{n})$ \\
Suspender todas as medidas de suporte & $4,3(05)$ \\
Acionar o neurologista para realizar o $2^{\circ}$ exame* & $81,7(94)$ \\
Manutenção do suporte avançado de vida* & $58,3(67)$ \\
Comunicar a família sobre a possibilidade do & $84,3(97)$ \\
diagnóstico de ME* & \\
Comunicar a família sobre a possibilidade de & $66,1(76)$ \\
doação de órgãos & \\
Chamar a equipe de transplante do hospital & $22,6(26)$ \\
Comunicar a central de órgãos & $40,0(46)$ \\
Colher exames para sorologia & $44,3(51)$ \\
\hline Condutas após confirmação do diagnóstico de ME & $\%(n)$ \\
Acionar a família para possível doação de ór- & $84,8(95)$ \\
gãos & $61,1(69)$ \\
Comunicar ao médico assistente* & $79,6(90)$ \\
Comunicar à família* & $63,7(72)$ \\
\hline Comunicar a central de órgãos* &
\end{tabular}

${ }^{*}$ Conduta considerada correta em cada momento.

Quando questionados sobre qual a importância de se realizar o diagnóstico de ME, 94,6\% $(n=106)$ dos entrevistados responderam para a doação de órgãos, $73,2 \%(n=82)$ para otimizar a rotatividade dos leitos de UTI, 68,8\% ( $n=77)$ para reduzir custos e $64,3 \%(n=72)$ para diminuir o sofrimento da família. Em relação aos fatores que mais dificultam o diag- 
nóstico de ME, 65,5\% ( $n=74)$ responderam que são os aspectos éticos, morais, religiosos e espirituais, $54 \%(n=61)$ os conhecimentos técnico-científico, $43,4 \%(n=49)$ as limitações institucionais, 33,6\% ( $=38)$ os aspectos legais e $13,3 \%(n=15)$ motivação financeira.

Apenas 15\% ( $n=17)$ dos estudantes entrevistados já avaliaram um paciente com ME. Destes, 52,9\% $(n=9)$ participaram da identificação do potencial candidato ao diagnóstico de ME, 58,8\% $(n=10)$ participaram da realização do protocolo, $35,3 \%(n=6)$ na manutenção de paciente com ME estabelecida e apenas 5,9\% (n =1) no contato, orientação e apoio à família. Os estudantes que realizaram algum estágio em UTI haviam avaliado pacientes com ME com maior freqüência quando comparados aos que nunca fizeram $(26,5 \%$ versus $6,3 \% ; p=0,003$ ), sendo essa diferença maior ainda quando foram avaliados apenas os estudantes que realizaram estágio de plantão $(38,2 \%$ versus $5,1 \% ; p<0,001)$.

Do total dos estudantes entrevistados, $92 \%(n=103)$ referiram que acreditam ou confiam no diagnóstico de ME. A maioria dos estudantes $(78,8 \% ; n=89)$ são doadores de órgãos, mas apenas $61,1 \%(n=69)$ comunicaram a sua decisão de ser ou não doador para a família. Dentre os não-doadores, o principal motivo apontado foi não confiar no sistema de saúde brasileiro $(58,3 \% ; n=14)$, seguido de questões filosóficas ou religiosas ( $25 \% ; n=6)$ e não confiar nos critérios diagnósticos de ME $(8,3 \% ; n=2)$.

\section{DISCUSSÃO}

Os resultados do presente estudo apontaram um nível de conhecimento relativamente baixo sobre o protocolo de diagnóstico de ME entre os estudantes de Medicina avaliados, com média de acerto de 6,7 em 14 questões. Outros autores já haviam relatado que os futuros médicos apresentam um conhecimento sobre ME inferior ao que é esperado. Essman e Thornton recentemente avaliaram 500 estudantes de Medicina do $1^{\circ}$ e $2^{\circ}$ anos da faculdade de Ohio (Estados Unidos) e relataram que o conhecimento sobre ME dos estudantes de Medicina era inferior ao de uma amostra de adultos da mesma região selecionados aleatoriamente, sendo esta diferença estatisticamente significativa ${ }^{10}$. Vale ressaltar ainda que os estudantes avaliados no presente estudo compõem uma população selecionada, por estarem participando de um evento científico extracurricular sobre Medicina Intensiva, são alunos di- ferenciados e particularmente interessados nos temas dessa área, podendo apresentar conhecimento superior à população acadêmica geral.

Este baixo nível de conhecimento se deve, em parte, ao pouco contato que os estudantes têm com este assunto durante a graduação. Neste trabalho, os estudantes que já tinham assistido alguma apresentação sobre o tema tiveram maior índice de acerto nas questões, demonstrando que maior ênfase, administrada na faculdade, poderia aumentar o nível geral de conhecimento dos estudantes. Ainda neste trabalho, $71 \%$ dos estudantes já haviam assistido alguma apresentação sobre ME, enquanto que apenas $15 \%$ já tinham avaliado algum paciente nesta condição. Nas duas faculdades avaliadas, o tema ME é abordado em apenas uma aula, na disciplina de Ética Médica e Bioética no $2^{\circ}$ semestre da escola privada e na disciplina de Clínica Cirúrgica no $8^{\circ}$ semestre da escola pública. Dutra e col. demonstraram que $96 \%$ dos estudantes avaliados, na mesma escola pública deste trabalho, gostariam de saber mais sobre a doação de órgãos e morte encefálica ${ }^{9}$.

A baixa importância atribuída a ME nas faculdades se repete para todos os temas relacionados à Medicina intensiva, como demonstrado em estudo anterior, realizado pela Liga Acadêmica de Medicina Intensiva da Bahia (LAMIB), que evidenciou que os estudantes apresentam grande interesse sobre estes temas, mas tem contato limitado com eles durante a graduação, principalmente na parte prática ${ }^{13}$. A realização de estágio em UTI também pode contribuir para maior contato dos estudantes com pacientes em ME, como demonstrado neste estudo. Nesta amostra, assim como no estudo já citado ${ }^{14}$, a maioria dos estudantes que fizeram estágio em UTI, o fizeram de forma extracurricular.

Os estudantes demonstraram maior número de acertos nas questões relacionadas aos aspectos teóricos do protocolo de ME, como critérios para suspeitar de ME e quais os profissionais envolvidos na avaliação. No entanto, nas questões relacionadas aos aspectos práticos, como os exames clínicos e complementares e as condutas relativas às diversas etapas do processo diagnóstico, os estudantes apresentaram uma média de acerto inferior. Dados semelhantes foram encontrados por Afonso e col. que avaliaram 580 estudantes de Medicina de diversos semestres em São Paulo e demonstraram que apesar de $70 \%$ dos estudantes conhecerem o conceito de ME, apenas $35 \%$ sabiam como fazer o diagnóstico ${ }^{6}$. 
O diagnóstico de ME é de extrema importância no contexto da terapia intensiva. Primeiramente, devido à necessidade de aumentar o número de doações e transplante de órgãos, sendo este o grande motivador do diagnóstico de ME. Além disso, o diagnóstico de ME em não doadores é fundamental para limitar o investimento em pacientes críticos, que na realidade já estão mortos, diminuindo assim o sofrimento da família e otimizando a rotatividade dos leitos, principalmente no sistema público. No entanto, existe grande resistência, por parte dos estudantes avaliados, em suspender o suporte artificial de manutenção dos órgãos, frente a um paciente não-doador com diagnóstico de ME. Em estudo realizado no México, em 1997, avaliando 114 médicos generalistas, especialistas e estudantes de Medicina, encontrou que $37 \%$ dos entrevistados acreditavam que os pacientes com diagnóstico de ME deveriam continuar recebendo tratamento intensivo até ocorrer a parada cardíaca ${ }^{15}$. Já na Polônia, em 1996, num estudo que avaliou o conhecimento sobre doação de órgãos de 1010 médicos generalistas, neurocirurgiões e anestesistas, 926 enfermeiras de UTI ou neurocirurgia e 1760 estudantes de medicina do país, foi encontrado que $100 \%$ dos médicos e $80 \%$ dos estudantes e enfermeiras aceitavam o conceito de ME, no entanto, apenas $44 \%$ dos médicos estavam preparados para desligar os apareIhos após o diagnóstico de ME em não-doadores ${ }^{16}$. Estes dados evidenciam a dificuldade dos profissionais de saúde em aceitar o diagnóstico de ME como morte clínica.

Apesar da grande maioria dos estudantes acreditarem no diagnóstico de ME, apenas $78,8 \%$ referiram ser doador de órgãos e um número menor $(61,1 \%)$ comunicou sua decisão a família. Por se tratar de uma amostra selecionada de indivíduos, com especial interesse em Medicina Intensiva, poderia ser esperado um percentual maior de doadores. No entanto, trabalhos anteriores também evidenciaram baixa freqüência de doadores entre os futuros médicos. Em um trabalho realizado no Japão, Ohwaki e col. encontraram que apesar dos estudantes de Medicina demonstrarem maior conhecimento sobre ME, quando comparados a outros estudantes universitários, não houve diferença entre os grupos em relação à doação de seus próprios órgãos ou de familiares ${ }^{12}$. Burra e col., na Itália, reportaram que a maioria dos estudantes que tinham atitudes negativas em relação a doação dos órgãos expressava dúvidas acerca do diagnóstico de $\mathrm{ME}^{17}$. Neste estudo, a principal justificativa dos não doadores foi não acre- ditar no sistema de saúde brasileiro.

Além da subnotificação dos pacientes com ME, a recusa familiar é uma das principais causas para o número relativamente reduzido de transplantes realizados no Brasil. A família apresenta grande relevância no processo diagnóstico, devendo ser informada sobre todos os passos do protocolo, assim como deve ser facultada a presença do médico de confiança para acompanhar a evolução. Após confirmação da ME e esclarecimento da família do processo de doação e benefícios do ato, cabe a ela decidir se os órgãos serão ou não doados. No entanto, ao contrário do que foi respondido pela maioria dos estudantes avaliados, deve ficar claro que o médico intensivista, que está participando do protocolo para o diagnóstico de $\mathrm{ME}$, não deve ser o responsável por abordar a família sobre a doação de órgãos. Esta atitude, principalmente antes da confirmação do diagnóstico, pode despertar a desconfiança da família e prejudicar o processo de doação. Por este motivo, a central de órgãos existe e deve ser acionada, após a confirmação diagnóstica, para tratar deste tema com a família e providenciar todos os procedimentos necessários para a doação.

Existem ainda muitas dúvidas acerca do tema ME, principalmente por ser um conceito novo, pouco difundido na população em geral e que não têm sido priorizado na graduação e pós-graduação. Na opinião dos estudantes avaliados os principais fatores que dificultam o diagnóstico são os aspectos éticos, morais e religiosos, seguidos de baixo conhecimento técnico-científico por parte dos médicos. Os primeiros estão relacionados a opiniões e crenças individuais, mas que muitas vezes estão embasadas em informações equivocadas sobre o tema. Gallagher, em 1996, avaliou a opinião de religiosos sobre a doação de órgãos e relatou que existe um entendimento errado do conceito de ME nessa população, sugerindo a necessidade de medidas educativas específicas nesta área ${ }^{18}$. DuBois e Anderson, numa revisão recente sobre estudos que avaliaram atitudes sobre os critérios de morte e doação de órgãos, mostraram que a população em geral e uma parte dos profissionais de saúde não estão familiarizados com os aspectos médicos e legais da $\mathrm{ME}^{19}$. Num trabalho realizado com médicos intensivistas baianos, os principais fatores apontados que dificultam o diagnóstico foram limitações institucionais e baixo conhecimento técnico-científico ${ }^{20}$. Estes resultados foram confirmados em estudo nacional que sugeriu que a estrutura hospitalar muitas vezes é inadequada para o diagnóstico de ME e que este 
protocolo ainda não está bem consolidado na prática diária dos intensivistas avaliados ${ }^{5,21}$.

Este estudo se diferencia dos demais por ser o primeiro a avaliar especificamente o conhecimento de estudantes de Medicina sobre o protocolo de ME. Não encontramos nenhum outro trabalho na literatura nacional ou internacional com esta característica. O estudo deste tema na graduação deve ser considerado como uma prioridade por permitir que o médico recém-formado que irá trabalhar em serviços de emergência ou UTI possa fazer este diagnóstico, atuando corretamente frente a estes pacientes. Além disso, independente de trabalhar em UTI ou emergência, os estudantes de Medicina, assim como os médicos e outros profissionais de saúde, devem ser referência para a comunidade na divulgação do conceito de ME e, conseqüentemente, contribuir para uma maior aceitação da população em relação a este diagnóstico e para um aumento no número de transplantes de órgãos.

\section{CONCLUSÃO}

O conhecimento dos estudantes de medicina avaliados em Salvador-BA sobre os critérios de diagnóstico da ME é limitado. Neste trabalho, encontrou-se que esta dificuldade está relacionada principalmente à abordagem prática do tema, como os exames clínicos e complementares a serem realizados e as condutas a serem tomadas em cada fase do protocolo diagnóstico. Foi observado que a participação em apresentações sobre ME e a realização de estágios em UTI podem contribuir, respectivamente, para um melhor conhecimento teórico e prático do tema. Estes achados destacam a necessidade de rever o currículo das escolas médicas com o objetivo de ampliar o conhecimento sobre o protocolo diagnóstico de ME, otimizando o processo de doação de órgãos. No entanto, mais estudos são necessários para definir quais fatores influenciam a aceitação da ME e a decisão de doar os órgãos para transplante.

\section{REFERÊNCIAS}

01. Wijdicks EFM - The diagnosis of brain death. N Engl J Med, 2001;344:12151221.

02. Parizi RR, Silma NM - Transplantes, em: Costa SIF, Garrafa V, Oselka G - Iniciação à Bioética. Brasília, Conselho Federal de Medicina, 1998;157-169.

03. Diagnosis of brain death: statement issued by the honorary secretary of the Conference of Medical Royal Colleges and their Faculties in the United Kingdom on 11 October 1976. Br Med J, 1976;2:1187-1188.

04. Guidelines for the determination of death. Report of the medical consultants on the diagnosis of death to the President's Commission for the Study of Ethical Problems in Medicine and Biomedical and Behavioral Research. JAMA, 1981;246:2184-2186.

05. Agareno S, Sousa RM, Santana DLP et al - Nível de conhecimento dos médicos intensivistas a respeito do protocolo de morte encefálica. RBTI, 2006;18:(Suppl.):P132.

06. Afonso RC, Buttros DA, Sakabe D et al - Future doctors and brain death what is the prognosis? Transplant Proc, 2004;36:816-817.

07. Bagheri A, Tanaka T, Takahashi $\mathrm{H}$ et al - Brain death and organ transplantation: knowledge, attitudes, and practice among Japanese students. Eubios J Asian Int Bioeth, 2003;13:3-5.

08. Bardell T, Hunter DJ, Kent WD et al - Do medical students have the knowledge needed to maximize organ donation rates? Can J Surg, 2003;46:453-457.

09. Dutra MM, Bonfim TA, Pereira IS et al - Knowledge about transplantation and attitudes toward organ donation: a survey among medical students in northeast Brazil. Transplant Proc, 2004;36:818-820.

10. Essman $\mathrm{C}$, Thornton $\mathrm{J}$ - Assessing medical student knowledge, attitudes, and behaviors regarding organ donation. Transplant Proc, 2006;38:2745-2750.

11. Laederach-Hofmann $\mathrm{K}$, Gerster $\mathrm{BI}$ - Knowledge, attitude and reservations of medical students about organ transplantation: results of a survey during the first year of study. Schweiz Med Wochenschr, 1998;128:1840-1849.

12. Ohwaki K, Yano E, Shirouzu M et al - Factors associated with attitude and hypothetical behaviour regarding brain death and organ transplantation: comparison between medical and other university students. Clin Transplant, 2006;20:416-422.

13. Bitencourt AGV, Almeida AM, Albuquerque LC et al - Medicina intensiva e o estudante de Medicina: interesses e contato durante a graduação. Rev Bras Educ Med, 2006;30:(Suppl.2):175.

14. Albuquerque LC, Almeida AM, Bitencourt AGV et al - Intensive care medicine teaching in a Brazilian medical school: the student's perspective. Crit Care, 2005;9:(Suppl2):P109.

15. Hernandez-Arriaga JL, Morales-Estrada A, Cortes Gallo G - Survey of physicians' attitudes to terminal patients. Rev Invest Clin, 1997;49:497-500.

16. Rowinski W, Ostrowski K, Adadynski L et al - Factors limiting renal transplantation program in Poland. Ann Transplant, 1996;1:18-22.

17. Burra $\mathrm{P}, \mathrm{De}$ Bona $\mathrm{M}$, Canova $\mathrm{D}$ et al - Changing attitude to organ donation and transplantation in university students during the years of medical school in Italy. Transplant Proc, 2005;37:547-550.

18. Gallagher C - Religious attitudes regarding organ donation. J Transpl Coord, 1996;6:186-190.

19. DuBois JM, Anderson EE - Attitudes toward death criteria and organ donation among healthcare personnel and the general public. Prog Transplant, 2006;16:65-73.

20. Agareno $\mathrm{S}$, Almeida $\mathrm{AM}$, Maia $\mathrm{J}$ et al - Opinião de médicos baianos sobre fatores dificultadores do diagnóstico de morte encefálica. RBTI, 2006;18:(Suppl):O66.

21. Agareno S, Soares MS, Santana DLP et al - Condições disponíveis nas unidades de terapia intensiva para realização do protocolo de morte encefálica. RBTI, 2006;18:(Suppl.):P109. 